\title{
La solución de un problema matemático no convencional por estudiantes universitarios
}

\author{
The solution of an unconventional mathematical problem by university students
}

\section{A solução de um problema matemático não convencional por estudantes universitários}

\author{
Juan Arturo Hernández-Morales ${ }^{1}$ \\ Apolo Castañeda ${ }^{2}$ \\ Rosa Isela González-Polo ${ }^{3}$
}

Recibido: noviembre de 2018

Aceptado: abril de 2019

Para citar este artículo: Hernández-Morales, J.A.; Castañeda, A.; González-Polo, R.I. (2019). La solución de un problema matemático no convencional por estudiantes universitarios. Revista Científica, 35(2), 201-215. Doi: https:// doi.org/10.14483/23448350.14863

\section{Resumen}

En la enseñanza tradicional, los problemas matemáticos suelen plantearse como una forma de aplicar conceptos o ejercitar procedimientos. Sin embargo, en esta investigación se propuso replantear este enfoque al introducir a la clase de matemáticas otros tipos de problemas para propiciar nuevas tareas y reflexiones. En el artículo se reporta la implementación de un problema matemático no convencional con el objetivo de identificar y analizar los argumentos de estudiantes universitarios. Este tipo de problemas se caracteriza por plantear consignas abiertas y admitir diversos procedimientos para su solución. Para analizar las producciones de los estudiantes, se utilizó el modelo argumentativo de Toulmin, el cual permitió identificar la estructura del razonamiento, desde los datos hasta la conclusión. Los resultados mostraron que algunos estudiantes enfrentaron dificultades debido a que la información en el problema no es del todo explícita y alegaron la falta de datos o errores en su redacción. En el caso de aqueIlos estudiantes que lograron resolverlo, se observó que se involucraron ampliamente con el problema, analizaron y expusieron diversas interpretaciones, se propició la discusión de las variables y sus relaciones y fueron utilizados conocimientos matemáticos para justificar sus procedimientos.

Palabras clave: didáctica, resolución de problemas, argumentación, matemática educativa, matemáticas escolares.

Universidad Politécnica de San Luis Potosí, México. arturo535@gmail.com

Departamento de Investigaciones Educativas (DIE-CINVESTAV), Centro de Investigación y de Estudios Avanzados del Instituto Politécnico Nacional, México apolo.castaneda@cinvestav.mx

3. Programa de Matemática Educativa, Centro de Investigación en Ciencia Aplicada y Tecnología Avanzada del Instituto Politécnico Nacional, México rgonzalezp1206@alumno.ipn.mx 


\section{Abstract}

In traditional education, mathematical problems are usually considered as a way of applying concepts or exercising procedures, however, in this research it was proposed to rethink this approach by introducing other types of problems to the mathematics class to encourage new tasks and reflections. The article reports the implementation of a non-conventional mathematical problem with the objective of identifying and analyzing the arguments of university students. This type of problems is characterized by open slogans and admit various procedures for their solution. To analyze the students' productions, the argumentative model of Toulmin was used, which allowed to identify the structure of the reasoning, from the data to the conclusion. The results showed that some students faced difficulties because the information in the problem is not completely explicit and they argued the lack of data or errors in its writing. In the case of those students who could solve it, it was observed that they were widely involved with the problem, they analyzed and presented different interpretations, the discussion of variables and their relationships was encouraged, mathematical knowledge was used to justify their procedures. Keywords: didactic, problem solving, argumentation, educational mathematics, school mathematics.

\section{Resumo}

Em problemas de ensino de matemática tradicionais, muitas vezes surgem como uma forma de aplicar os conceitos ou procedimentos de exercícios, no entanto, esta pesquisa foi a repensar esta abordagem a través da introdução de aula de matemática outros problemas para promover novas tarefas e reflexões. O artigo relata a implementação de um problema matemático não convencional com o objetivo de identificar e analisar os argumentos de estudantes universitários. Esse tipo de problema é caracterizado por slogans abertos e admitem vários procedimentos para sua solução. modelo argumentativo de Toulmin, o que nos permitiu identificar a estrutura de raciocínio de dados para a realização foi utilizado para analisar produções estudantis. Os resultados mostraram que alguns alunos enfrentaram dificuldades porque as informações do problema não são completamente explícitas e argumentaram a falta de dados ou erros em sua escrita. Para os alunos que se pudessem resolvê-lo, notou-se que o problema envolveu extensivamente analisados e apresentados diferentes interpretações, a discussão das variáveis e suas relações são promovidas, o conhecimento matemático foi usado para justificar seus procedimentos.

Palavras-chaves: didático, resolução de problemas, argumentação, matemática educacional, matemática escolar.

\section{Introducción}

Aunque la resolución de problemas matemáticos es un tema que ha sido ampliamente estudiado (Armella y Santos-Trigo, 2013), esta investigación tiene el propósito de reflexionar en torno a la implementación un problema matemático, al que Schoenfeld (1985) denomina "problema no rutinario", con la intención de realizar una valoración cualitativa de los argumentos que los estudiantes exponen durante su proceso de resolución (Calderón, 2006). Este tipo de problema no rutinario se caracteriza por plantear situaciones que no tienen estructura convencional a los que presentan usualmente en un curso de matemáticas; caso contrario, estos emplean un contexto amplio y, en ocasiones, la información no aparece del todo explícita, por lo que se requiere hacer interpretaciones, inferencias e, incluso, realizar una reflexión previa para identificar una cuestión central. Cuando los estudiantes se enfrentan a este tipo de problemas suelen tener dificultades, ya que no se parecen a los típicos problemas escolares en los que los datos aparecen bien identificados y que para resolverlos basta con elegir la fórmula, realizar la operación y escribir el resultado. La introducción de problemas no convencionales al aula puede constituir un recurso natural para motivar la discusión, la exposición de conjeturas, contraejemplos y aproximaciones (Santos, 2007), así como el intercambio y enfrentamiento de puntos de vista.

De acuerdo con Cerbin (1998), la actividad de resolver problemas se apoya de las 
argumentaciones para justificar procedimientos y resultados, pero esta relación se vuelve más estrecha cuando se abordan problemas con una estructura abierta o problemas no convencionales (Cho y Jonassen, 2002), ya que este tipo de problemas admiten diversas interpretaciones y procedimientos de solución. Los típicos problemas matemáticos con una estructura definida no ofrecen un amplio margen para explorar, discutir o proponer estrategias debido a lo predecible y mecánico que resulta su proceso de solución, por lo que los argumentos en estos casos suelen fundamentarse únicamente en los algoritmos que le dan una validez al procedimiento y a la solución. En cambio, la introducción de problemas no rutinarios permite que los estudiantes se involucren en una amplia reflexión sobre los elementos planteados, no solo respecto a la información matemática en juego, sino también los aspectos del contexto del propio problema.

Dado que la resolución de problemas plantea un contexto de aprendizaje en el cual los argumentos contribuyen a formular ideas y repensar los problemas (Lester, 2013; Cerbin, 1988), se planteó el estudio de la implementación de un problema matemático no rutinario, así como el análisis de los argumentos que exponen estudiantes universitarios durante su solución (Schoenfeld, 1985), el propósito es distinguir las características de los argumentos particularmente cuando establecen el procedimiento de solución.

\section{Marco conceptual}

De acuerdo con Schoenfeld (1985), la definición de problema admite diversas interpretaciones debido a que se trata de algo relativo; lo que para una persona requiere de un esfuerzo significativo, para otra puede representar un ejercicio de rutina. Pero, afirma que un problema es una relación particular entre el individuo y la tarea, específicamente cuando la tarea representa un desafío y no se conoce el esquema de solución. El individuo debe emplear sus recursos matemáticos para establecer un posible procedimiento a partir de la información proporcionada en el problema, esto implica reconocer su estado inicial e identificar las condiciones y características del resultado final.

La habilidad para resolver problemas es reconocida como una forma de razonamiento de alto nivel (Santos, 2007) que involucra el uso y desarrollo de un pensamiento no algorítmico (Arcavi y Friedlander, 2002), así como la articulación de una variedad de acciones cognitivas que involucran conocimientos y habilidades específicas, algunas de ellas no rutinarias, tales como elegir las representaciones apropiadas, establecer conjeturas, coordinar experiencias y conocimientos previos, incluso desarrollar una intuición matemática para generar procedimientos e integrar algoritmos (Lester, 2013).

En este sentido, Lester propone un modelo para describir el proceso de resolución de problemas en el que se destaca el tránsito entre dos mundos, el cotidiano de problemas y el abstracto de conceptos, símbolos y operaciones matemáticas. Así, los elementos más importantes son las relaciones entre el proceso matemático (mundo matemático) y las acciones sobre los elementos particulares del problema (mundo cotidiano). En su modelo, Lester (2013) describe los momentos por los que transita un individuo que trabaja en la solución de un problema matemático, el cual es entrelazado y multifase, ya que considera que el actuar de un individuo en el proceso de solución, puede requerir de una constante comparación y esta se puede presentar en las diversas fases, modificando su actuar en el proceso de solución que desarrolla el individuo.

Este esquema inicia con un contexto en el cual se le propone al estudiante a trabajar en la resolución de un problema $(A)$, se le plantea una consigna específica que debe atender (flecha sólida entre $A$ y $B$ ). El estudiante simplifica el planteamiento al identificar conceptos y procesos que soportan al problema $(B)$. El estudiante vuelve al contexto inicial para reinterpretar en sentido del problema (flecha punteada entre $B$ y $A$ ). A continuación, viene la fase de abstracción (flecha sólida entre $B$ y $C$ ) la 


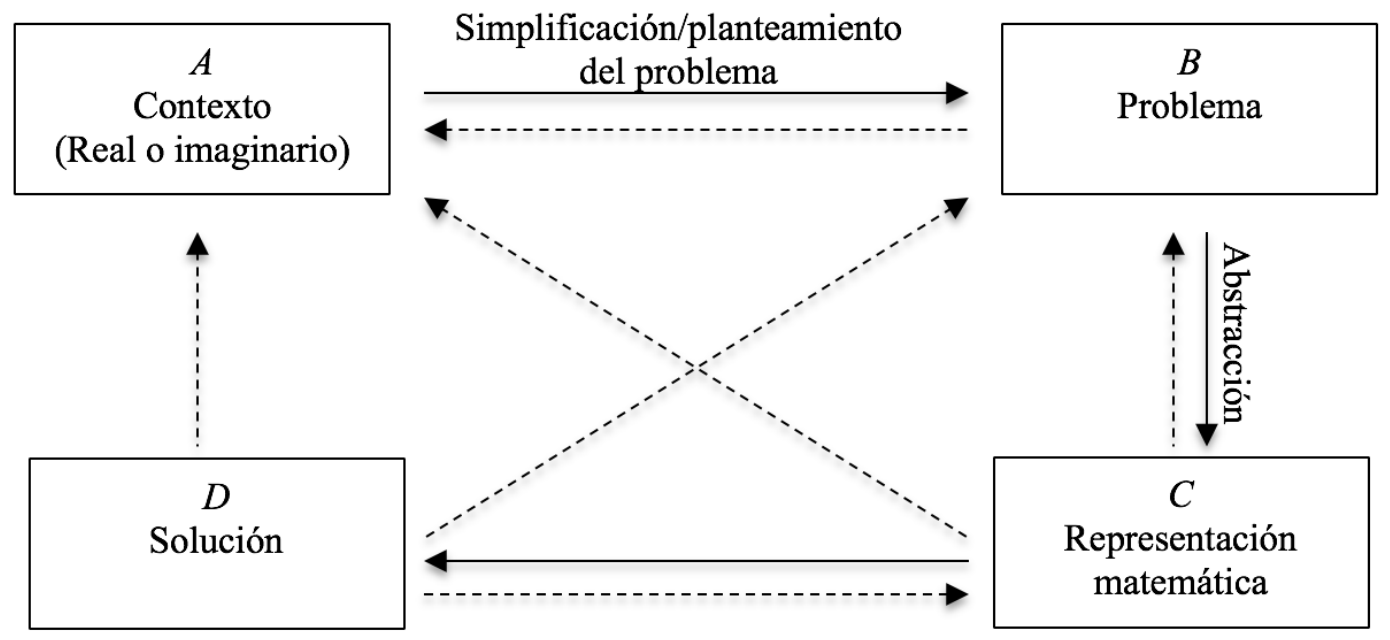

Figura 1. Modelo de actividad matemática de resolución de problemas.

Fuente: traducción propia a partir de Lester (2013).

cual introduce conceptos matemáticos y notaciones (idiosincrásicas). Esta fase de abstracción involucra la selección de conceptos matemáticos que representen las características esenciales del modelo. Una vez que el estudiante ha generado una representación matemática de la situación original, este problema matemático adquiere un significado propio, volviéndose un problema matemático aislado, bien definido (cuadro C). La tercera fase del proceso (de $C$ a $D$ ) involucra la manipulación de la representación matemática y la deducción de algunas conclusiones matemáticas (señaladas como la flecha sólida de cálculos). Por último, la fase, que puede ser de $D$ a $A, D$ a $B$, y $D$ a $C$, implica la comparación de las conclusiones o resultados con el problema en su contexto original o con su representación matemática. Esta etapa no implica necesariamente la conclusión del ciclo, ya que se trata de un proceso de continuo de monitoreo, la cual es definida por Lester como una actividad metacognitiva de reflexión continua, que representa un elemento clave para el éxito en tareas matemáticas complejas. Lester afirma que el grado con el cual un individuo compara el estado actual del problema con estados anteriores denota la complejidad de la tarea, permitiendo distinguir así tareas rutinarias o no rutinarias. Esta investigación pone especial atención a lo que ocurre en el momento de la "abstracción" en el modelo anterior, ya que en esta fase el estudiante elige e introduce conceptos matemáticos, notaciones esenciales para resolver el problema y aparecen argumentaciones que conducen a la solución de problema.

\section{Argumentación}

Metaxas, Potari y Zachariades (2016) señalan que la argumentación contribuye a la construcción de conocimiento, pues implica el uso de habilidades para justificar, desafiar, contraponer o conceder ideas respecto de un conocimiento. No obstante, como lo afirman Inglis y Mejía-Ramos (2005), es común que los estudiantes universitarios presenten dificultades al formular y sustentar sus argumentos en el contexto de una actividad matemática. Determinar el origen de esas dificultades ha motivado el estudio y análisis de las argumentaciones para describir la naturaleza de los argumentos, los elementos que los sustentan, así como su función en el desarrollo de una actividad matemática. El aula de clase representa el contexto idóneo para realizar observaciones de los procesos argumentativos. En este ámbito se puede analizar el trabajo de los alumnos para identificar el papel que tienen 
las explicaciones, afirmaciones, cuestionamientos en el estudio de conceptos matemáticos. Tal como lo señalan De Gamboa, Planas y Edo (2010), la argumentación es un discurso que se dirige a un receptor con el fin de justificar una opinión a partir de hechos, razonando los criterios sobre los que se decide la adecuación de la opción elegida. Sin embargo, no todas las actividades que se realizan en clase permiten a los alumnos exponer públicamente los argumentos, heurísticas y conjeturas que elaboran durante en sus actividades matemáticas. Pedirle a un estudiante exponer explícitamente sus argumentos no siempre resulta fácil debido a la ausencia de la actividad metareflexiva en los procesos matemáticos que realiza; además, como afirman Kidron y Dreyfus (2010), incluso los estudiantes de nivel universitario carecen de la claridad conceptual para formular o enunciar un argumento matemático. Esta situación alentó el desarrollo de este trabajo, al proponer un problema para el cual los estudiantes se involucren en el diálogo, debate y puedan exponer sus puntos de vista, sostener sus ideas y construir argumentos. De Gamboa, Planas y Edo (2010) señalan que la argumentación se desarrolla como parte de la actividad matemática en donde las "razones" se proponen como "justificación" entre los datos y la conclusión, aunque estas proposiciones deben ser de naturaleza deductiva y no solo semántica. De esta forma, el estudiante podría reconocer el poder que tienen sus argumentos en el desarrollo de sus propias conclusiones, las cuales contribuyen a fundamentar su aprendizaje.

La identificación de argumentos matemáticos se puede realizar utilizando el esquema argumentativo de Toulmin (Inglis, Mejía-Ramos y Simpson, 2007), el cual está conformado por seis tipos de declaraciones y cada una de estas cumple un papel diferente en el argumento. Inglis y Mejía-Ramos (2005) plantean el modelo en la figura 2.

Inglis y Mejía-Ramos (2005) señalan que la conclusión (C) es la tesis que se invoca; el dato (D) es la información en la cual se basa la conclusión que está formada por hechos o condiciones que son observables; la justificación (J) es una declaración que justifica la conexión entre el dato y la conclusión, haciendo referencia, por ejemplo, a una regla, definición, analogía, que brinda la lógica para la transición del dato a la conclusión, además, justifica la importancia del dato. La "justificación" es el apoyo o respaldo que puede ser un estudio, un código, una estadística o incluso una creencia. El calificador (Q) determina la fuerza de la conclusión, en dicha declaración se expresa el grado de confianza en la tesis. Y la refutación (R) presenta las excepciones de la conclusión, es decir, las condiciones en las cuales no es posible sostener la tesis del argumento.

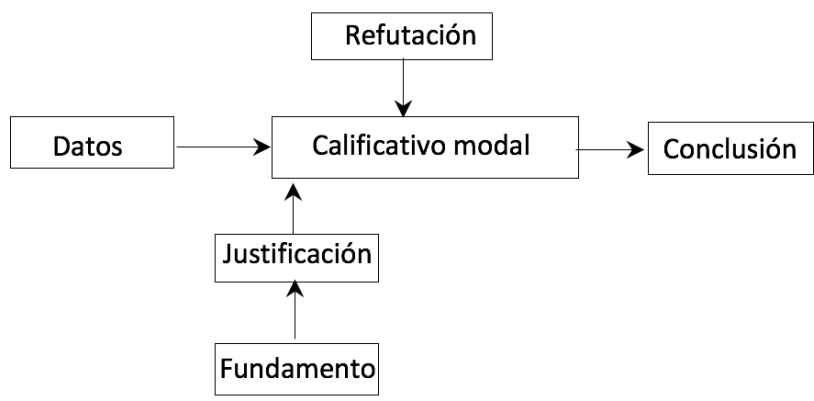

Figura 2. Esquema del modelo de Toulmin.

Fuente: traducción propia a partir de Inglis y MejíaRamos (2005).

Inglis y Mejía-Ramos señalan que no todos estos tipos de declaraciones son necesariamente verbalizados de manera explícita por los estudiantes durante una actividad matemática, lo que dificulta su identificación. Dado que la resolución de problemas es un tipo de actividad matemática que demanda la puesta en funcionamiento de diversos recursos matemáticos, habilidades, estrategias de solución y que, además, favorece un contexto de diálogo, debate y argumentación, esta investigación postula plantear una extensión del modelo de Lester (2013) e integrar al momento de "abstracción" el modelo argumentativo de Toulmin, ya que en ese momento los estudiantes también elaboran argumentos, implícitos o explícitos, y es donde justifican sus decisiones y procedimientos. 


\section{Metodología}

Para llevar a cabo la investigación, primero se formuló un problema no rutinario (Schoenfeld, 1985) distinto a los resueltos comúnmente en clase de matemáticas, ya que, como señalan Alvarado y González (2009), el proceso habitual de instrucción en matemáticas no ofrece oportunidades para que los alumnos expresen verbalmente sus impresiones; además de que los problemas que se ponen en marcha son muy estructurados, con información precisa, reglas claras y criterios definidos para resolverlos (Simon, 1973). En cambio, los problemas no rutinarios admiten varios procedimientos de solución, en el que se requiere, en conjunto conlas reglas, fórmulas y algoritmos, el uso de conjeturas, contraejemplos y aproximaciones (Santos, 2007). Considerando lo planteado por Schoenfeld (1985), se formuló un problema denominado "Vuelo a Madrid", que está inspirado en un itinerario real de viaje. El problema plantea determinar el tiempo de viaje y la diferencia de horarios entre las dos ciudades, en el problema se indican los horarios de salida y llegada en la hora local de la ciudad correspondiente.

La actividad se aplicó en tres grupos de estudiantes universitarios en la modalidad de trabajo en equipo, uno de primer semestre (grupo 1) que cursaban la materia Introducción a las matemáticas; un grupo de estudiantes de tercer semestre que cursaban la materia Cálculo diferencial e integral (grupo 2); y otro de séptimo semestre que cursaban la materia Métodos numéricos (grupo 3), con un total de 58 estudiantes, todos eran alumnos de la carrera de ingeniería en sistemas computacionales en una universidad pública de la ciudad de San Luis Potosí, en el estado del mismo nombre en México. La selección de estos grupos atendió al criterio de integrar una muestra con estudiantes de cursos básicos, intermedios y avanzados. El profesor titular del curso (el mismo en los tres grupos) fue el responsable de poner en marcha la actividad durante el horario escolar. Los estudiantes del primer grupo (básico) habían cursado asignaturas de matemáticas básicas, como álgebra y trigonometría. Los estudiantes del segundo grupo (intermedios) habían cursado geometría analítica, álgebra lineal, probabilidad, estadística. Los estudiantes del tercer grupo (avanzados) habían cursado variable compleja y ecuaciones diferenciales.

En la primera fase, los estudiantes trabajaron en equipo en la solución del problema, después se realizó una sesión grupal para analizar los resultados. Durante las jornadas de trabajo se utilizó un equipo de filmación para recuperar las interacciones verbales y se recopilaron los registros escritos de cada estudiante con el propósito de analizar e interpretar los planteamientos matemáticos.

\section{Problema "Vuelo a Madrid"}

\section{Indicaciones}

Haz una justificación y descripción lo más detallada que puedas, tanto del procedimiento desarrollado como de tus resultados, argumenta lo más ampliamente posible las conclusiones derivadas de estos.

\section{Vuelo a Madrid}

Iván viaja en avión desde la Ciudad de México a Madrid, el avión sale de la Ciudad de México el lunes a las 6:25 p.m. (hora local) y llega a Madrid el martes a las 12:40 p.m. (hora local). Después de pasar una semana de trabajo viaja de regreso, sale de Madrid el lunes a las 2:40 p.m. (hora local) y llega a la Ciudad de México el lunes a las 7:20 p.m. (hora local), Iván notó que el viaje de regreso tardó 25 minutos más que el de ida.

\section{Preguntas}

Con los datos que se tienen en el enunciado anterior: ¿es posible determinar cuánto tiempo dura el viaje de México a Madrid?, ¿es posible determinar cuánto tiempo hay de diferencia en los horarios entre estas ciudades? 
Para el diseño del problema se consideró: 1) plantear un contexto que permitiera una fácil interpretación entre los estudiantes; 2) el uso de consignas abiertas; (3) atendiendo a las características de los problemas "no rutinarios", se consideró importante no hacer explícitos todos los datos de la situación a fin de motivar el intercambio de puntos vista; y 4) permitir a los estudiantes el uso de cualquier recurso matemático para su solución. Además, en el ejercicio, el profesor evitó cualquier intervención a fin de no influenciar el proceso de solución. Atendiendo al modelo de Lester (2013), se centró la atención en la fase de "abstracción", que se caracteriza por el uso de conceptos matemáticos y notaciones que representan los elementos del modelo real, los cuales conducen al desarrollo de una representación matemática de la situación planteada. En esta fase se observa el uso de recursos propios de los estudiantes, así como los argumentos para justificar la elección del procedimiento. Por esta razón, se procuró que el planteamiento del problema no indujera un procedimiento matemático específico y permitiera formular diversas rutas de solución.

\section{Procedimientos de solución esperados}

Existen al menos tres procedimientos para resolver el problema: empleando operaciones aritméticas, a través de un sistema de ecuaciones de $2 \times 2$ o través de una ecuación lineal de primer grado. Presentamos a continuación la solución del problema empleando un sistema de ecuaciones.

En cualquier viaje, el tiempo de duración se determina a través de una diferencia entre la hora de salida y llegada al destino. En el problema identificamos como elemento central la hora de salida y llegada en cada ciudad, ya que a partir de estos datos es posible determinar el tiempo de vuelo aparente, es decir, el tiempo de vuelo sin considerar las horas de diferencia entre las ciudades. El vuelo de la Ciudad de México a Madrid sale el lunes a las 6:25 pm y llega el martes a las 12:40 pm, existe una diferencia de 18 horas 15 minutos, es decir,
1095 minutos en el tiempo aparente de vuelo. El vuelo de Madrid a la Ciudad de México sale el lunes a las 2:40 pm y llega el lunes a las 7:20 pm, existe una diferencia de 4 horas con 40 minutos, es decir, una diferencia de 280 minutos en el tiempo aparente de vuelo. Se observa que existe una diferencia considerable entre los tiempos de vuelo calculados anteriormente, la cual puede ser explicada por la diferencia de horarios. En el primer viaje se agrega al tiempo aparente de vuelo, las horas de la diferencia horaria; por el contrario, al regreso el tiempo de vuelo se restan las horas de diferencia horaria. En el vuelo de la Ciudad de México a Madrid, representamos con $v$ el tiempo aparente de vuelo y d las horas de diferencia entre estas ciudades, tenemos:

$$
v+d=1095
$$

Sabemos que en el vuelo de Madrid a la Ciudad de México duró 25 minutos más, por lo que el tiempo de vuelo lo representamos como $v+25$ y las horas de diferencia $\mathrm{d}$ se restan, entonces tenemos:

$$
v+25-d=280
$$

Esto conduce a un sistema de dos ecuaciones con dos variables:

$$
\begin{gathered}
v+d=1095 \\
v+25-d=280
\end{gathered}
$$

Aplicando eliminación por suma o resta tenemos:

$$
\begin{gathered}
2 v+25=1375 \\
v=675
\end{gathered}
$$

Por lo cual:

$$
d=420
$$

Con lo cual concluimos que existen siete horas de diferencia entre ambas ciudades. 


\section{Cómo resolvieron el problema}

\section{Propuestas de solución grupo 1}

Las soluciones planteadas por el grupo 1 son aritméticas; en ningún caso se observó un planteamiento algebraico o el uso de variables. Los cálculos realizados giran en torno a las diferencias de horas entre los vuelos. Se observa también una gran cantidad de errores en los cálculos aritméticos. En el planteamiento de uno de los estudiantes Ilamado Elder se señala que existe un error en los datos del problema y esto lo lleva a resultados inconsistentes y da por concluida su solución. Dice que:

Se tarda 18 hrs con 5 min en su viaje de ida y de su regreso llegó de Madrid a México en solo 6 hrs, no es posible y aun así menciona que se tardó 25 minutos más que la ida, el problema no está planteado correctamente o con datos erróneos porque no es posible que haya 12 hrs de diferencia en ida y llegada, debe ser más o menos igual.

De acuerdo con el esquema de Lester (figura 1), identificamos que este grupo de estudiantes tiene dos vertientes en el grado de la comparación en la etapa de solución; aquellos que identificaron mediante una diferencia la duración de los vuelos (18 horas con 15 minutos y 4 horas con 40 minutos) y aquellos que responden basándose en los tiempos dados y no hacen una comparación final, ni se preguntan por la congruencia de los resultados obtenidos. Los estudiantes que señalan una "inconsistencia" en los datos del problema desarrollaron un proceso de comparación más profundo que quienes no lo observaron.

\section{Propuestas de solución grupo 2}

Las soluciones del grupo 2 son muy parecidas a las reportadas por el grupo 1. La mayoría de los estudiantes plantearon el cálculo de diferencias del tiempo transcurrido en los que se observaron varios errores aritméticos. Sin embargo, una diferencia sustancial que se observó en este grupo fue la formulación de explicaciones para las aparentes inconsistencias relativas en los tiempos de vuelo, atribuidas al contexto de un viaje, tales como cambios de horario, retraso de los vuelos, cambio de rutas entre los viajes. Por ejemplo, en el planeamiento de una de las estudiantes Ilamada Jazary señala que:

Hay muchos factores que están inmersos aquí, como las posibles escalas, aduanas, retraso en los vuelos, mal clima, transbordos, cambios de avión a último minuto (depende de la aerolínea).

Un elemento distintivo de este grupo de estudiantes fue la aparición de notación algebraica en el planteamiento, aunque sin éxito. Por ejemplo, otro estudiante Ilamado Bernardo planteó ecuaciones formuladas a partir de los horarios, donde reconoce que el tiempo de vuelo de ida es $x$ y el regreso $x+25$, pero son ecuaciones que no le conducen al resultado esperado.

De acuerdo con esquema de Lester (Figura 1) este grupo involucra los aspectos contextuales del problema de forma más explícita en su proceso de solución, ya que se observan gráficos, dibujos, figuras, e incluso aparecen personajes de historieta que hablan sobre cómo pudo haber ocurrido, que derivó en la afectación del tiempo de vuelo. Esto fue motivado por la comparación (señalado en la figura 1) entre el modelo matemático y los datos del problema.

\section{Propuestas de solución grupo 3}

En el caso de los estudiantes de séptimo semestre observamos que son pocos los que señalan que el problema está mal redactado. En este grupo es común el planteamiento de tipo algebraico a través de un sistema de ecuaciones, en el que identifican la variable $x$ asociada al tiempo de vuelo y la y asociada a la diferencia de horarios entre las ciudades. En este grupo aparecen procesos matemáticamente exitosos en la justificación de 
resultados. Los estudiantes se dan cuenta de la aparente inconsistencia de los tiempos de vuelo, pero la mayoría la explican a partir de la diferencia de horarios. Por ejemplo, en el caso del estudiante Ilamado Christian, señala que el problema puede conducir a afirmaciones erróneas, incluso él explica la causa de esta situación:

[...] podríamos decir que el viaje dura menos la segunda vez, pero en realidad debes darte cuenta en el tiempo real que dura el viaje, o sea, el tiempo en el que el avión está en el aire.

En síntesis, podemos destacar que el problema matemático que se planteó a los estudiantes, al menos para los grupos 1 y 2, representó un problema en el sentido de Lester (2013) y Schoenfeld (1985). Se esperaba que los estudiantes mostraran un amplio dominio matemático en la solución; sin embargo, las dificultades reportadas hacen constatar que esta tarea representó un fuerte desafío. Se encontraron pocas soluciones correctas en los estudiantes de los grupos 1 y 2 . En el grupo 2 se observaron varias justificaciones que resolvían la aparente inconsistencia en el problema, agregando información adicional para tratar de encontrar una solución. En el caso de los estudiantes del grupo 3, se observó un mejor desempeño en la resolución del problema, particularmente al incorporar deducciones algebraicas y apoyándose únicamente de los datos señalados en el problema. Varios estudiantes comentaron que la actividad no representó gran dificultad. Para el análisis de los argumentos reportados en este artículo, se consideraron las producciones de los estudiantes del grupo 2, debido a que, para los estudiantes del grupo 1 resultó ser una actividad muy compleja, varios de los estudiantes advirtieron una "inconsistencia" pero la mayoría siguió estrictamente el planteamiento del problema y respondieron sin considerar pertinentes las diferencias en el tiempo de vuelo. Por otra parte, para los estudiantes del grupo 3 no representó un reto y lograron identificar y explicar las aparentes inconsistencias, dando una solución muy consistente al problema.

\section{Discusión de resultados: los momentos en los que aparecen los argumentos}

Para determinar la duración de los vuelos la mayoría de los estudiantes del grupo 2 planteó la diferencia entre el horario de salida y de llegada. Esto los Ilevó a concluir que el tiempo de duración de ambos vuelos no es igual y ante este hecho aparecen dos tipos de argumentos: aquellos que atribuyen situaciones propias del contexto del problema que no están explícitas en el problema, como suponer una demora de uno de los vuelos; o bien, aquellos que señalan un error en el planteamiento. A continuación, se presenta el análisis realizado a tres diálogos que se desarrollaron en el primer momento de trabajo en equipo con estudiantes del grupo 2. Como se ha señalado, los estudiantes trabajaron en equipo y estuvieron acompañados por el profesor en breves periodos de tiempo, debido a que el docente tenía que recorrer todos los equipos del grupo. En las intervenciones, el profesor tuvo el papel de orientar el diálogo y motivar la exposición de argumentos.

Para realizar este análisis se utilizó el modelo de Toulmin descrito en Inglis y Mejía-Ramos (2005). La secuencia de la argumentación consiste en demostrar la conclusión a partir de los datos. Esta relación se expresa en la línea principal del trazado y puede referirse a la inferencia del argumento, pero esa inferencia requiere una legitimidad (respaldos), que representan fundamentos básicos indiscutibles (justificación, fundamentos). Como lo señalan Teixeira, Freire y Greca (2015), la estructura de un argumento se puede resumir de la siguiente forma: J, una vez aprobado por $\mathrm{F}$, autoriza a C, obtenida a partir de D, siendo considerado $\mathrm{Q}$, a menos que haya $\mathrm{R}$.

Los argumentos pueden ser encadenados de tal manera que una conclusión puede funcionar de nuevo como dato para un nuevo argumento posterior (Krummheuer, 2015). En nuestro caso, los argumentos se refieren al planteamiento del proceso de solución y esto concluye con un resultado obtenido, sea correcto o incorrecto. De acuerdo con 
Inglis y Mejía-Ramos (2005), no todos los tipos de declaraciones son necesariamente verbalizados de manera explícita, de ahí la importancia de la intervención del profesor para motivar a los estudiantes a exponer sus ideas.

Para este reporte se presentan tres diálogos, que fueron seleccionados considerando la amplitud y claridad de las declaraciones de los estudiantes. Los dos primeros desarrollados entre el profesor $(\mathrm{P})$ y los estudiantes del equipo 4, Bernardo (B), Karina (K) y Anahí (A). El tercer diálogo se establece entre $\mathrm{P}$ con el equipo 3 conformado por Martín (M), Ludivina (L) y Semiramis (S). Al momento de realizar esta conversación los estudiantes ya habían superado la fase de abstracción, así que ya habían acordado el procedimiento que desarrollarían en la fase de representación matemática. El primer diálogo se refiere a la conclusión "el problema se resuelve con una derivada" expuesta por Bernardo.

\section{Episodio 1: Bernardo y el profesor}

P: Y bien ¿cómo se resuelve el ejercicio?

B: Yo creo que el tiempo... de la hora de salida de México a la hora de llegada, es algo así como una resta es igual a $x$, y después la hora de salida de Madrid a la hora de llegada igual una resta... pero sería equis más veinticinco $(x+25)$ minutos que es lo que dice que demoró.

P: ¿Y usaste ese razonamiento para la solución?, o lo estás pensando ahora.

B: No, pues yo le puse que se hacía con una derivada, porque es una razón de cambio las horas... de México y de Madrid.

P: ¿Qué está cambiando?

B: Pues es diferente la hora de aquí (México) a la de allá (Madrid), ¿no?

P: ¿Es un variable?

B: (asiente).

P: ¿Y la otra? en la razón de cambio involucramos dos variables, ¿ verdad?

B: Pues el tiempo.

P: ¿Es la hora?

B: (asiente).
P: Ok, pero tú dices que tienes esa incógnita que es la equis $x$, ¿ es el tiempo verdad?, y de regreso equis más veinticinco $x+25$.

B: Sí, pero podría ser una ecuación.

A partir de este diálogo se construyó el esquema de la figura 3, en el que se observa que la idea de "diferencia" fue la que motivó la asociación con la derivada y, por lo tanto, afirmó que se trataba de un tema de cálculo diferencial. El argumento expuesto por Bernardo estuvo influido por un contrato didáctico (en el sentido de Brousseau), ya que en días previos a la aplicación de la actividad se abordó el tema de "derivada" en el curso de cálculo.

\section{Episodio 2: Anahí y el profesor}

El segundo diálogo se refiere a la conclusión "el problema no se puede resolver" que expone Anahí.

P: La primera pregunta que les voy a hacer es ¿cómo se resuelve el problema?

A: Bueno a mí se me hizo un problema muy difícil porque yo sentía que nos faltaban datos para poderlo resolver.

P: De acuerdo, ¿qué fue lo que pensaste, Anahí?

A: Bueno pues yo empecé restando como si fueran las horas iguales, las de aquí (México) a las horas de allá (Madrid) yo puse que en el primero eran 18 horas y 15 minutos y en el segundo eran 4 horas y 40 minutos, entonces yo concluí que no era posible, por razonamiento lógico no era posible determinar el tiempo ya que los datos no eran los... que ocupábamos para poder resolver, pues cuando uno viaja el tiempo de viaje es lo primero que se sabe.

$\mathrm{P:}$ ¿Qué concluyes?

A: No se puede resolver, está mal planteado.

A partir de este diálogo se construyó el esquema de la fgura 4. Anahí señala que la diferencia obtenida en los vuelos no era congruente, ya que, de acuerdo con su experiencia de viaje, esos datos son precisos, por lo tanto, no es posible resolver el problema. 


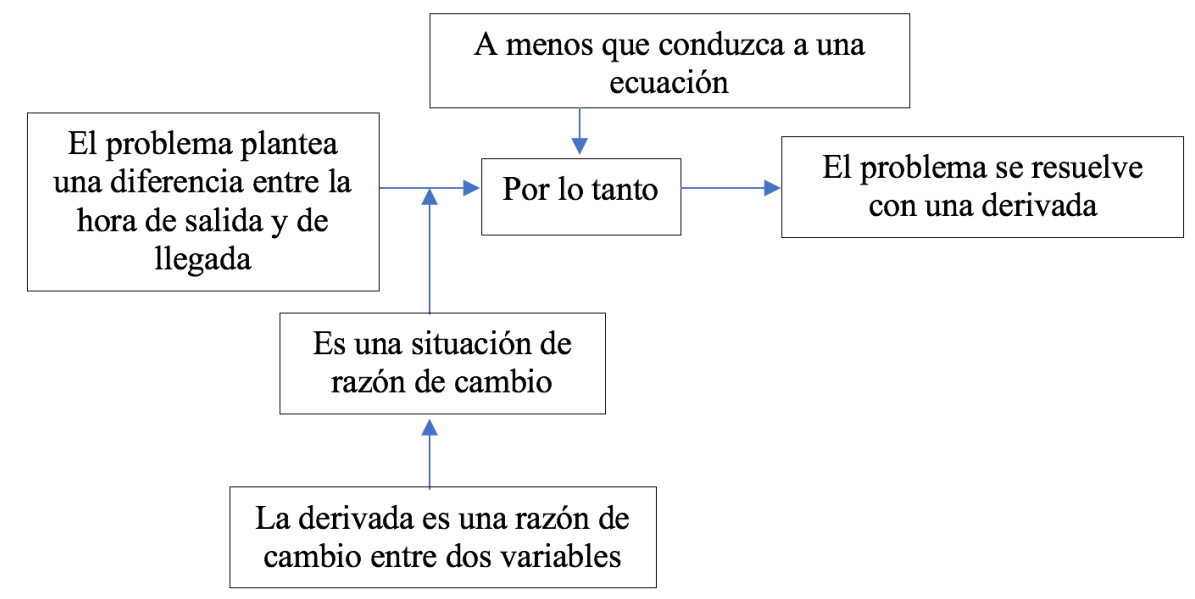

Figura 3. Esquema para el argumento de Bernardo.

Fuente: elaboración propia.

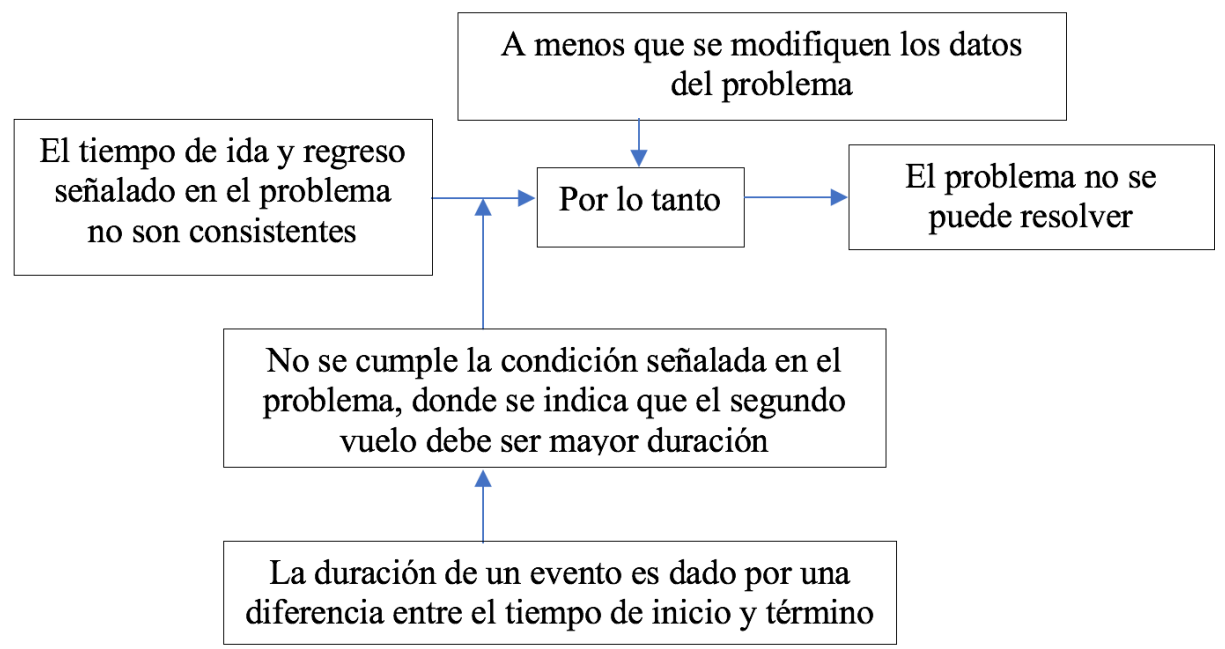

Figura 4. Esquema de argumentos de Anahí.

Fuente: elaboración propia.

\section{Episodio 3: Martín, Ludivina, Semiramis y el profesor}

El tercer diálogo se refiere a la conclusión "el problema se resuelve a través de un sistema de ecuaciones".
P: ¿Con los datos que tienen es posible determinar el tiempo de viaje de México a Madrid?

L: Sí.

P: ¿Cómo se podría hacer eso?

L: Mediante una ecuación.
P: De acuerdo.

S: Ya lo intentamos.

M: Un sistema de ecuaciones.

P: ¿Y con eso será posible determinar la diferencia de horarios?

M: Sí

L: Yo digo que no, pero...

P: Ella dice que no, tú dices que sí, ¿ por qué lo afirmas Martín?

M: Porque nos da dos datos diferentes, antes pensaba diferente, pero ahora que lo analizo de esta manera nos conduce a un sistema 
de ecuaciones. Entonces podemos determinar algo, ya sea que encontremos las horas que tarda en viajar o la diferencia de horario si hacemos correctamente el sistema de ecuaciones podemos encontrar uno de estos.

P: Tú dices que si encuentras una variable después ya puedes encontrar la otra.

M: En el sistema de ecuaciones, ese es ahí donde yo creo que está, nos está faltando definir.

P: ¿Qué opinan ustedes muchachas?, ¿será correcto lo que dice su compañero?, ¿están de acuerdo?

L: Bueno sí, sí ya encontramos una variable ya nada más sería de despejar y ya podríamos encontrar la otra.

A partir del diálogo anterior se elaboró una línea argumentativa en la figura 5. En este episodio, Martín señala que el problema tiene dos incógnitas, por lo que esto puede dar lugar al planteamiento de un sistema de ecuaciones. Conociendo el valor de una de las variables, entonces se puede determinar la otra. El problema es establecer esta relación de variables y emplear un método para resolver el sistema.

El profesor encargado de la implementación recorrió todos los equipos para entablar conversación, con el propósito de obtener información sobre la forma en que cada equipo planteó la solución del problema. Esta se organizó y se formularon cuatro categorías atendiendo a la forma en que cada equipo planteó la solución al problema, estas categorías se describen a continuación.

a. El problema no se puede resolver debido a que faltan de datos (C). Los estudiantes identifican en el problema las horas de salida y Ilegada (D) y plantean operaciones para calcular la diferencia de tiempo $(\mathrm{F})$, y determinar el tiempo de vuelo de cada viaje (J), pero al realizar las operaciones obtienen resultados que no se ajustan a las condiciones del problema y admiten que son inconsistentes por lo que (Q) no se puede resolver a menos que se agreguen información adicional $(\mathrm{R})$ al problema para subsanar los huecos de información, por ejemplo, aclarar cuántas escalas hizo el avión o la velocidad del avión en cada viaje.

b. No se puede resolver el problema debido a errores en la redacción (C), los estudiantes identifican las horas de salida y llegada (D) y determinan que el problema se resuelve con una resta $(F)$, lo que permite determinar la duración del tiempo de vuelo (J), pero al plantear y resolver sus operaciones obtienen resultados que no concuerdan con la situación planteada y admiten que sus resultados son inconsistentes y no son lógicos con lo que ocurre en la

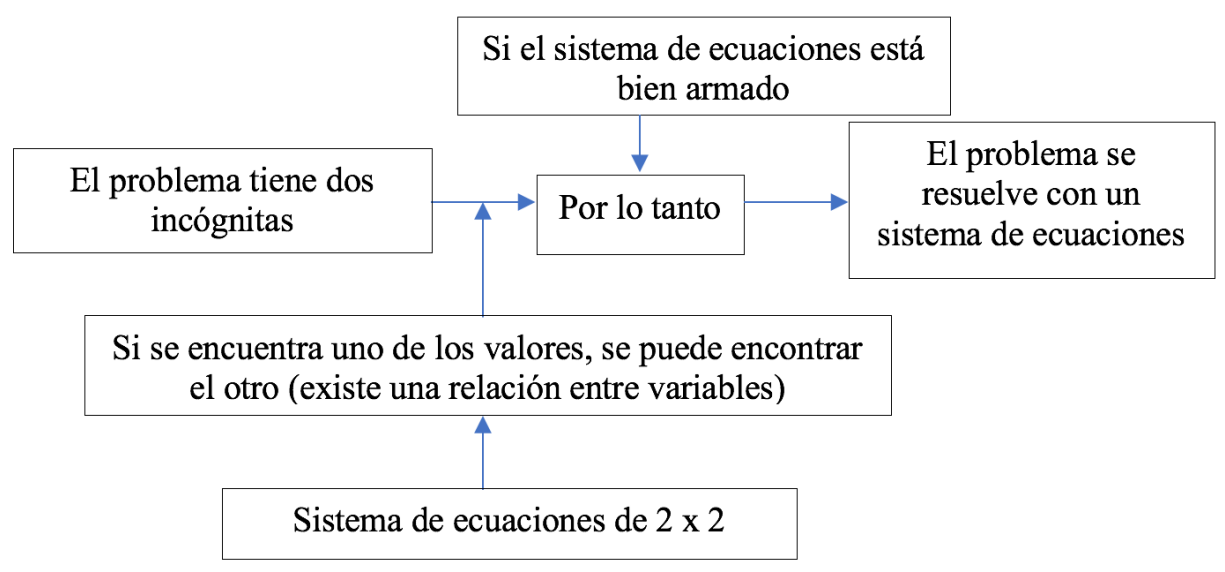

Figura 5. Esquema de argumentos de Martín.

Fuente: elaboración propia. 
vida real. A menos que se modifique el planteamiento y establezcan correctamente los datos (R).

c. El problema se puede resolver a través de una operación o ecuación (C). Los estudiantes identifican la hora de salida y de llegada (D) y establecen operaciones o plantean ecuaciones (F) que permitirán determinar el tiempo de vuelo (J). Después de efectuar el procedimiento matemático señalado, se obtiene un resultado equivocado, que no se confrontan con el planteamiento y se acepta como válido. No emplean un procedimiento para verificar sus resultados y confían en el resultado de la operación. Las excepciones (R) son de dos tipos. La primera referida a la posibilidad de plantear o usar una operación o ecuación incorrecta. La segunda, la posibilidad de un error de cálculo que conduzca a un resultado equivocado.

d. Homólogo al anterior, con la diferencia de que las operaciones o ecuaciones planteadas conducen a la solución correcta, sin que necesariamente todos hayan Ilegado a ella. En el caso de aquellos estudiantes que obtuvieron un resultado, lo validan al sumar la diferencia horaria al tiempo de vuelo de cada viaje.

Estas categorías antes descritas ponen de manifiesto que los datos del problema establecen el punto de partida para el proceso de solución y definen la conclusión a partir de tres referentes matemáticos: 1) los conocimientos que han abordado o estudiado previamente en su curso de matemáticas, bajo la idea de que el problema planteado es un ejercicio de "aplicación"; (2) el planteamiento y ejecución de cálculos matemáticos y ecuaciones simples, sin una reflexión previa sobre el uso de esos recursos matemáticos y asumiendo que los problemas se resuelven aplicando ciertas operaciones; y 3) la identificación de variables en el problema y la asociación inmediata a la formulación de ecuaciones para determinar su relación, tanto en ecuaciones lineales como sistemas de ecuaciones. Las categorías a y $b$ exponen los casos donde se afirma que el problema no se puede resolver, en estos, la "excepción" permite a los estudiantes justificar una modificación al problema: ajustar su redacción para eliminar inconsistencias o bien para agregando información complementaria para hacerlo más preciso.

La categoría $c$ se refiere al caso en el que los estudiantes no realizan una reflexión sobre el sentido del problema durante la fase de la abstracción, y continúan con la solución sin preguntar la coherencia de sus resultados. La justificación se sostiene en la idea de diferencia entre horarios como forma de determinar la duración del viaje, y el fundamento en las operaciones aritméticas. Así, los estudiantes no expresan ninguna dificultad y confían en que su procedimiento los llevará a la solución correcta del problema. Este fenómeno, denominado "contrato didáctico", se refiere a comportamientos específicos de los estudiantes cuando responden a un planteamiento matemático para cumplir con lo que, creen, se espera de ellos (Education Committee of the European Mathematical Society, 2012). Uno de estos casos es un problema que se presenta bajo el nombre de "la edad del capitán", el cual enuncia: "en un barco hay 26 ovejas y 10 cabras, ¿Cuál es la edad del capitán?", las investigaciones que exploraron las respuestas de este problema (como la de Verschaffel, Greer y de Corte, 2000) señalan que la gran mayoría de los alumnos realizan cálculos para determinar un valor a través de alguna operación como suma o resta, sin que sea cuestionada la estructura del problema. Es decir, confían en que el profesor ha asignado un problema correctamente ya que sería extraño que un ejercicio no tuviera solución.

\section{Conclusiones}

Una de las características de los problemas "no convencionales" es que los datos no son explícitos, están incompletos y en ocasiones no hay una respuesta única al problema. Ante esto, los estudiantes dedican más tiempo a la interpretación de la 
situación, la deducción de datos y a la construcción de procedimientos para resolver el problema. Esto suele generar incertidumbre ya que los estudiantes deben tomar decisiones sobre todas las etapas en la solución del problema, no obstante, esto representa un importante potencial didáctico al permitir a los estudiantes poner en juego sus recursos matemáticos, explorar diferentes rutas de solución y propiciar la comunicación de información matemática con sus compañeros.

Usualmente, los libros de texto abordan muy pocos problemas de este tipo, y más bien se enfocan en plantear problemas que asocien o favorezcan el uso de ciertos procedimientos, técnicas o métodos.

Sin embargo, es importante enfatizar que esta distinción en los tipos de problemas no es del todo absoluta pues, como lo señala Schoenfeld (1985), existe la posibilidad de que un mismo planteamiento implique diferente esfuerzo aún para estudiantes con los mismos antecedentes matemáticos. Postulamos la idea de que los problemas "no convencionales" tienen ciertos rasgos distintivos que posibilitan la creación de un ambiente para explorar, debatir y justificar los procedimientos de solución. No obstante, esto debe tomarse con cautela pues en esta investigación se observó que algunos estudiantes, incluso trabajando en equipo, no cuestionaron ni reflexionaron sobre el sentido de sus operaciones y los resultados obtenidos. Este grupo de estudiantes presentó sus resultados sin que realizaran un enfrentamiento con el contexto y las condiciones señaladas en el problema. Otro grupo de estudiantes (avanzados), no reveló ninguna dificultad la solución del problema pues, para ellos, resultó muy sencillo. Tampoco se generó mucha interacción ya que planteamiento surgió rápidamente y no hubo conflictos. Otro grupo de estudiantes consiguió abordar el problema como un desafío, en él apareció una constante reflexión sobre el sentido del problema y, aunque no todos Ilegaron a la solución completa, sí hubo justificaciones correctas sobre los planteamientos matemáticos elegidos. Este grupo fue el elegido para profundizar en el análisis de sus argumentos.
El problema creado para esta investigación tuvo la característica de emplear consignas abiertas, lo que permitió la formulación de diversas interpretaciones, que, incluso, pusieron en duda la estructura y coherencia del problema. Por otra parte, permitió el uso de cualquier recurso matemático para su solución, lo cual ofreció libertad a los estudiantes en la elección del procedimiento. Estas dos características, aunado a un esquema de trabajo colectivo para favorecer la interacción, motivaron que en la fase de "abstracción" se identificaran al menos cuatro diferentes conclusiones (C) asociadas a la solución del problema.

Este trabajo ofrece dos contribuciones al campo de la educación matemática, el primero al profundizar en la compresión y origen de los argumentos o razones lógicas de los estudiantes, a través del modelo argumentativo de Toulmin, durante la fase de "abstracción" en el modelo de resolución de problemas de Lester (2013). La conjunción de estas dos unidades teóricas resultó muy favorable pues permitió ordenar los recursos matemáticos y procedimientos que pusieron en juego los estudiantes para la solución del problema (observados en la fase de "abstracción") e identificar el origen de las conclusiones que formulan los estudiantes. La segunda contribución se refiere a una serie de reflexiones sobre las características de los procedimientos que eligieron los estudiantes para la solución del problema, directamente asociados a sus antecedentes matemáticos, pero también influidos por la interpretación del problema. Como se pudo observar, los estudiantes más jóvenes atribuyeron una supuesta inconsistencia del problema que limitó el trabajo matemático.

\section{Referencias}

Arcavi, A.; Friedlander, A. (2002), Curriculum developers and problem solving: the case of Israeli elementary school projects. ZDM, 39 (5-6), 5-22. https://doi.org/10.1007/ $\underline{\text { s11858-007-0050-3 }}$ 
Armella, L. M. y Santos-Trigo, M. (2013). Introduction to International Perspectives on Problem Solving Research in Mathematics Education. The Mathematics Enthusiast, 10 (1).

Alvarado, A. y González, M. T. (2009), La implicación lógica en el proceso de demostración matemática: estudio de un caso. Enseñanza de las ciencias, 28(1), 73-84.

Calderón, D.I. (2006). El análisis de tareas para el desarrollo de competencias argumentativas en geometría. Revista Científica, 8, (3), 185-202. https://doi.org/10.14483/23448350.344

Cerbin, B. (1988), The nature and development of informal reasoning skills in college students. Conferencia presentada en el National Institute on Isues in Teaching and Learning, Chicago, U.S.A.

Cho, K-L. y Honassen, D. (2002), The Effects of Argumentation Scaffolds on Argumentation and Problem Solving. Educational Studies in Mathematics 50 (3), 5-22. https://doi.org/10.1007/ BF02505022

De Gamboa, G., Planas, N. y Edo, M. (2010), Argumentación matemática: prácticas escritas e interpretaciones. Suma, 64, 35-44.

Education Committee of the European Mathematical Society (2012), What are the Reciprocal Expectations between Teacher and Students? Solid Findings in Mathematics Education on Didactical Contract. Newsletter of the European Mathematical Society, 84, pp. 53-55.

Inglis, M. y Mejía-Ramos, J. P. (2005), La fuerza de la aserción y el poder persuasivo en la argumentación en matemáticas. Revista EMA, 10 (3), 328-353.

Inglis, M., Mejía-Ramos, J. P. y Simpson, A. (2007), Modelling mathematical argumentation: the importance of qualification. Educational Studies in Mathematics, 66, 3-21. https://doi. org/10.1007/s10649-006-9059-8
Kidron, I. y Dreyfus, T. (2010), Justification enlightenment and combining constructions of knowledge. Educational Studies in Mathematics, 74, 75-93. https://doi.org/10.1007/ s10649-009-9228-7

Krummheuer, G. (2015), Methods for Reconstructing Processes of Argumentation and Participation in Primary Mathematics Classroom Interaction. En Bikner-Ahsbahs A., Knipping C., Presmeg N. (eds) Approaches to Qualitative Research in Mathematics Education. Advances in Mathematics Education. Springer, Dordrecht

Lester, F.K. (2013), Thoughts about research on mathematical problem solving instruction. The Mathematics Enthusiast, $10(1$ y 2), 245-278.

Metaxas, N. D., Potari, D. y Zachariades, T. (2016). Analysis of a teacher's pedagogical arguments using Toulmin's model and argumentation schemes. Educational Studies un Mathematics, 93 (3), 383-397. https://doi.org/10.1007/ s10649-016-9701-z

Santos-Trigo, L. M. (2007), La resolución de problemas matemáticos: fundamentos cognitivos. México: Trillas.

Schoenfeld, A. (1985), Mathematical problem solving. Orlando, FL: Academic Press.

Simon, H. A. (1973), The structure of ill-structured problems. Artificial Intelligence, 4, 181-201. https://doi.org/10.1016/0004-3702(73)90011-8

Teixeira, E., Freire, O. y Greca, I. (2015), La enseñanza de la gravitación universal de Newton orientada por la historia y la filosofía de la ciencia: una propuesta didáctica con un enfoque en la argumentación. Enseñanza de las Ciencias 33 (1), 205 -223

Verschaffel, L. G., Brian y De Corte, E. (2000), Making sense of word problems. The Netherlands: Swets \& Zeitlinger. 